\title{
Psychosis and aggression in Alzheimer's disease: the effect of dopamine receptor gene variation
}

\author{
C Holmes, H Smith, R Ganderton, M Arranz, D Collier, J Powell, S Lovestone
}

\begin{abstract}
This study investigated possible associations between selected polymorphisms in the dopamine receptor genes DRD1 and DRD3 with the presence of psychotic phenomena or aggressive behaviour in a community based cohort of 134 patients with late onset Alzheimer's disease. An association was found between the presence of psychotic symptoms and aggressive behaviour and the DRD1 polymorphism and between the presence of psychosis, but not aggression, and the DRD3 polymorphism. Specifically, carriers of the DRD1 B2 allele were more likely to be aggressive or experience hallucinations whereas homozygous carriers of the DRD3 1 allele were more likely to experience delusions. (F Neurol Neurosurg Psychiatry 2001;71:777-779)
\end{abstract}

Keywords: Alzheimer's disease; genetics; behaviour

Cognitive decline is, by definition, invariably present in Alzheimer's disease. However, various other behavioural and psychological symptoms in dementia (BPSD) are also present, with psychotic symptoms found in about a third of cases and aggressive behaviour in around a half. The mechanism by which BPSD occurs in some patients with Alzheimer's disease is not completely understood but we have previously shown that common genetic polymorphisms in the serotonin receptors 5-HT2A and 5-HT2C are associated with the presence of psychotic phenomena, particularly hallucinations, in Alzheimer's disease. ${ }^{1}$ More recently, a study of the dopamine DRD1, 2, 3, and 4 receptors ${ }^{2}$ has suggested an association between common polymorphisms in the DRD1 and DRD3 receptors and the presence of aggression and psychosis in Alzheimer's disease. This study was designed to examine the role that these polymorphisms might have on the development of specific components of psychotic phenomena-namely, the presence of delusions and hallucinations in patients with late onset Alzheimer's disease.

\section{Methods}

PATIENTS

Patients were selected from the Camberwell Dementia Case Register. This is a community based register consisting of patients referred to psychiatric, medical, and social services with the districts of East Lambeth and South Southwark (formerly Camberwell), London, from April 1993 to April 1995. All patients were screened using the minimental state examination (MMSE) and any patients scoring less than 24 points were included for further assessment. Of the 528 patients fulfilling these criteria 374 also fulfilled the National Institute of Neurological and Communicative Disorders and Stroke and the Alzheimer's Disease and Related Disorders Association criteria ${ }^{3}$ for possible and probable Alzheimer's disease of late onset. Of these patients, consent for DNA testing was available in 198, of whom 134 had late onset probable $\mathrm{AD}$. Clinical diagnoses have been previously validated against postmortem findings with high positive predictive values for the clinical diagnosis of probable $\mathrm{AD}$ against CERAD diagnostic criteria. ${ }^{4}$

The patients and the next of kin or main carer were interviewed in all cases by means of the CAMDEX ${ }^{5}$ and the presence of a psychiatric history defined according to these guidelines - that is, an emotional or nervous illness requiring treatment. Presence of psychopathology was assessed by the Manchester and Oxford Universities scale for the psychological assessment of dementia (MOUSEPAD). ${ }^{6}$ Due to possible ethnic differences in the frequencies of DRD1 and DRD3 receptor polymorphisms only white persons were included in this study. Sufficient genetic material was available for DRD3 genotyping in 129 cases and for DRD1 genotyping in 105 cases.

Ethical approval was obtained from the Bethlem Royal and the Maudsley Hospital and Institute of Psychiatry ethics committee.

DOPAMINE RECEPTOR GENETIC ASSAYS Genotyping for DRD1 and DRD3 was performed using specific polymerase chain reaction protocols as described elsewhere. ${ }^{78}$

\section{STATISTICAL ANALYSIS}

The frequency of symptoms in patients with the DRD 1 variants $\mathrm{B} 1 / 2$ and $\mathrm{B} 2 / 2$ and DRD 3 variants $1 / 2$ and $2 / 2$ was compared with the DRD1 variant B1/B1 and DRD3 variant $1 / 1$, respectively, using odds ratios with $95 \%$ confidence intervals (95\% CIs).

\section{Results}

The mean age at the time of initial presentation to the register was 83.5 years (SD 6.1) with a 
Table 1 Odds ratios of developing symptoms in patients with DRD1 B1/B2 and B2/B2 genotypes compared with B1/B1 genotype

\begin{tabular}{llllll}
\hline \multicolumn{5}{c}{} & \multicolumn{2}{l}{$\begin{array}{l}\text { Number of patients with } \\
\text { symptom }\end{array}$} & & & \\
\cline { 2 - 3 } DRD1 Genotype & Absent & Present & Odds ratio & $95 \%$ CI & p Value \\
\hline Aggression: & & & & & \\
$\quad$ BI/BI & 17 & 6 & 3.8 & $1.3-11.2$ & 0.014 \\
BI/B2 & 21 & 28 & 2.1 & $0.2-2.1$ & 0.209 \\
B2/B2 & 19 & 14 & & & \\
Delusions: & 13 & 10 & 2.2 & $0.8-6.1$ & 0.114 \\
BI/BI & 18 & 31 & 1.8 & $0.6-5.2$ & 0.299 \\
BI/B2 & 14 & 19 & & & \\
B2/B2 & 22 & 1 & 9.7 & $1.2-79.0$ & 0.006 \\
Hallucinations: & & 15 & 5.9 & $0.7-52.0$ & 0.067 \\
BI/BI & 34 & 7 & & & \\
BI/B2 & 26 & & & & \\
B2/B2 & & & & & \\
\hline
\end{tabular}

mean age of onset of 78.6 years (SD 6.3). The mean MMSE (SD) score was 11.2 (SD 6.7) with a mean duration of illness at the time of initial interview of 4.9 years (SD 3.3). Most $(n=103(77 \%))$ of the patients were women. Twenty two patients $(16.4 \%)$ had a psychiatric history. Of the 134 patients included in this study $59(44.0 \%)$ had exhibited aggressive symptoms, $32(24.0 \%)$ hallucinations, and 78 $(58.2 \%)$ delusions. The presence or absence of BPSD within Alzheimer's disease was not influenced by the presence of a psychiatric history.

Frequencies of the DRD1 genotype for all patients according to the presence or absence of aggressive or psychotic phenomena are presented in table 1. Patients with Alzheimer's disease with the B1/B2 genotype were more likely to show aggressive behaviour than those with the B1/B1 genotype (odds ratio 3.8; $95 \%$ CI $1.3-11.2 ; \mathrm{p}=0.014)$. Subjects with a B1/B2 genotype were also more likely to experience hallucinations (odds ratio 9.7; 95\% CI 1.2$79.0 ; p=0.006)$ than $\mathrm{B} 1 / \mathrm{B} 1$ patients. Patients with the B2/B2 genotype showed a nonsignificant trend (odds ratio $5.9 ; 95 \%$ CI 0.7 to $52.0 ; \mathrm{p}=0.067$ ) towards an increase in hallucinations compared with patients with the B1/B1 genotype. No significant relations were found between DRD1 genotype and the presence or absence of delusions. Multivariable analysis with previous psychiatric history and DRD1 genotype as independent variables and the presence or absence of aggression or hallucinations as dependent variables did not alter these relations.

No significant relations were found between DRD3 genotype and the presence or absence of aggression or hallucinations. However, patients homozygous for the DRD3 2/2 allele were less likely to experience delusions than DRD3 1/1 patients (odds ratio 0.3; 95\% CI $0.1-0.99 ; p=0.045)$. However, this was not significant for DRD3 1/2 patients compared with DRD3 $1 / 1$ patients (odds ratio 0.8; 95\% CI $0.4-1.9 ; \mathrm{p}=0.764)$. Multivariate analysis with psychiatric history and DRD3 genotype as independent variables and the presence or absence of delusions as the dependent variable did not alter these relations.

\section{Discussion}

This study was restricted to patients with late onset Alzheimer's disease and this is reflected in the elderly nature of the group, with an advanced mean age and age of onset. The patients in this study had a relatively long duration of illness at the point of initial interview, which was shown by their relatively advanced disease status as assessed by the MMSE. These findings make it more likely that BPSD would have been expressed and thus documented than if caregivers had been interviewed at an earlier point in the course of the illness. The frequency of psychotic and aggressive symptoms found in this community based study falls within the ranges for disease lifetime prevalence found in other studies of Alzheimer's disease. ${ }^{9}$

The presence of a psychiatric history does not seem to influence the frequency of other psychotic symptoms or aggressive features found in patients with Alzheimer's disease. This finding suggests that most BPSD start during Alzheimer's disease and that these symptoms are intrinsic to or dependent on some aspect of the disease process.

These findings largely support those of an earlier study ${ }^{2}$ suggesting an association between variation in the DRD1 receptor gene with psychosis and aggressive behaviour and an association between variation in the DRD3 gene with psychosis, but not with aggression, in Alzheimer's disease. However, there are some differences in genotype and allele frequencies between symptomatic and asymptomatic patients. Specifically, earlier findings ${ }^{2}$ suggested that homozygosity for the DRD1 B2 allele was particularly associated with aggression or psychosis. In this study we found that the major effect was due to a lack of aggression or psychosis in patients who were homozygous for the DRD1 B1 allele. Thus, patients carrying one copy of the DRD1 B1 allele exhibited a greater frequency of psychotic symptoms or aggressive behaviour compared with noncarriers. As in earlier findings ${ }^{2}$ we found no association between variation in the DRD3 gene and the presence of aggression. However, unlike earlier findings suggesting that homozygosity for either DRD3 allele was associated with psychosis in $\mathrm{AD}$ we were unable to find any evidence of a general trend towards an increased frequency of psychotic symptoms in homozygotes.

The presence of a previous psychiatric illness had little influence on these genetic associations. These findings suggest that variation in the DRD1 gene, in the presence of coexistent $\mathrm{AD}$, can result in the development of psychotic symptoms (predominantly hallucinations) or aggressive behaviour. Likewise, variation in the DRD3 gene, shown in some studies ${ }^{8}$ to be associated with schizophrenia, may also be clinically silent in some patients but can result in the development of psychotic symptoms (delusions) which are only revealed by the subsequent development of $\mathrm{AD}$. 
1 Holmes C, Arranz MJ, Powell JF, et al. 5-HT2a and 5-HT2c receptor polymorphisms and psychopathology in late onset

2 Sweet RA, Nimgaonkar VL, Kamboh MI, et al. Dopamine receptor genetic variation, psychosis, and aggression in Alzheimer's disease. Arch Neurol 1998;55:1335-40.

3 McKhann G, Drachman D, Folstein M, et al. Clinical diagnosis of Alzheimer's disease: report of the NINCDS ADRDA Work Group under the auspices of Department of Health and Human Services Task Force on Alzheimer's Disease. Neurology 1994;34:939-44.

4 Holmes C, Cairns N, Lantos P, et al. Validity of current criteria for Alzheimer's disease, vascular dementia, and dementia with Lewy bodies. Br f Psychiatry 1999;174:45-50.

5 Roth M, Huppert F A, Tym E, et al. CAMDEX. The Cambridge examination for mental disorders of the elderly. Cambridge, UK: Cambridge University Press, 1988.
6 Allen NH, Gordon S, Hope T, et al. Manchester and Oxford Universities scale for the psychopathological assessment of dementia (MOUSEPAD). Br F Psychiatry 1996;169:293307.

7 Cichon S, Nothen MM, Stober G, et al. Systematic sceening for mutations in the 5'-regulatory region of the human dopamine receptor (DRD1) gene in patients with schizo1996;67:424-8.

8 Nimgaonkar VL, Sanders AR, Ganguli R, et al. Association study of schizophrenia and the dopamine D3 receptor gene locus in two independent samples. Am f Med Genet 1996; 67:505-14.

9 Cummings J, Miller B, Hill MA, et al. Neuropsychiatric aspects of multi-infarct dementia and dementia of the Alzheimer type. Arch Neurol 1987;44:389-93.

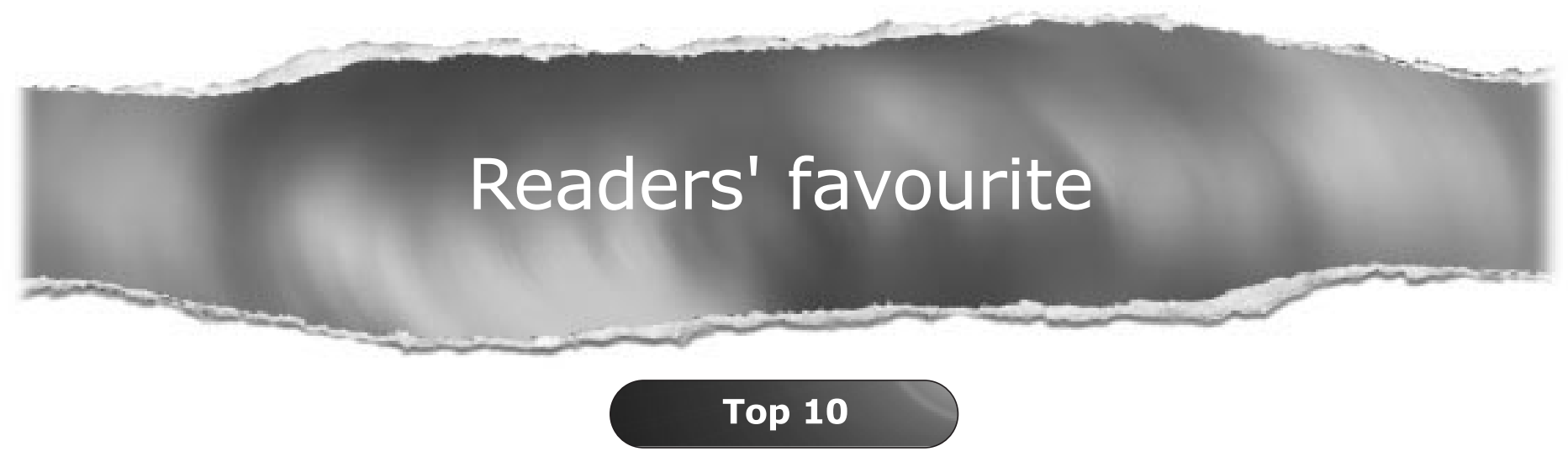

Click on the "Top 10" button on the homepage to see which are the best read articles each month

\section{www.jnnp.com}

